\title{
EFECTO DE UNA INTERVENCIÓN NUTRICIONAL INTENSIVA EN PACIENTES REFRACTARIOS A LA PÉRDIDA DE PESO
}

\section{RESUMEN}

Objetivo: Valorar el efecto de una intervención nutricional intensiva, basada en la aplicación de una Dieta Mediterránea hipocalórica personalizada, sobre el peso corporal y el perímetro de la cintura en adultos refractarios a la pérdida de peso.

Material y métodos: Se realizó un estudio prospectivo durante 6 meses en 100 participantes con edades comprendidas entre 55-75 años, IMC $\geq 27 \mathrm{y}<40 \mathrm{~kg} / \mathrm{m}^{2}$, que cumplieran con 3 o más criterios de síndrome metabólico. La intervención consistió en la pauta de una Dieta Mediterránea hipocalórica, recomendaciones de ejercicio y entrevistas motivacionales. Se recogieron variables antropométricas 1 vez al mes durante toda la intervención. Para analizar los resultados se realizaron los test de Chicuadrado y T-Student y se calculó mediante regresión logística no condicional los Odds Ratio con intervalos de confianza del $95 \%$ de las variables asociadas a la pérdida de peso.

Resultados: Tras la intervención de "rescate", los pacientes perdieron una media del $2,9 \%$ del peso corporal y del $2,1 \%$ del perímetro abdominal, alcanzando el $38 \%$ de la muestra una pérdida $\geq 3 \%$ del peso y el $26 \%$ de la muestra una reducción $\geq 3 \%$ de circunferencia de cintura. No se observaron diferencias estadísticamente significativas en la pérdida de peso y cintura en ninguna de las variables analizadas.

Conclusiones: Una intervención intensiva, basada en una Dieta Mediterránea hipocalórica, recomendación de actividad física y charlas motivacionales, consigue pérdidas moderadas de peso en pacientes refractarios a los tratamientos contra la obesidad.

Palabras clave: Dieta hipocalórica, Dieta Mediterránea, obesidad, pérdida de peso, tratamiento nutricional. 


\section{ABSTRACT}

Objective: To assess the effect of an intensive nutritional intervention, based on the application of a personalized hypocaloric Mediterranean diet, on the body weight and waist perimeter in refractory adults to weight loss.

Methods: A prospective study was conducted for 6 months in 100 participants with an age range between 55 and 75 years, $\mathrm{BMI} \geq 27$ and $<40 \mathrm{Kg} / \mathrm{m}^{2}$, which fulfilled 3 or more criteria of metabolic syndrome. The intervention consisted in the pattern of a hypocaloric Mediterranean diet, exercise recommendations and motivational talks. Anthropometric variables were collected once a month during the entire intervention. To analyze the results, the Chi-square and T-Student tests were performed and the Odds Ratio with confidence intervals of $95 \%$ of the variables associated with weight loss was calculated by non-conditional logistic regression.

Results: After the "rescue" intervention, the patients lost an average of $2.9 \%$ of the body weight and $2.1 \%$ of the abdominal perimeter, reaching $38 \%$ of the sample the target of the loss $\geq 3 \%$ of the weight and $26 \%$ of the

sample the target of reduction $\geq 3 \%$ of waist circumference. No statistically significant differences were observed in weight and waist loss in any of the variables analyzed.

Conclusion: Intensive intervention, based on a hypocaloric Mediterranean diet, recommendation of physical activity and motivational talks, achieve moderate weight loss in refractory patients to treatments against obesity.

Keywords: Hypocaloric diet; Mediterranean Diet; obesity; weight loss; nutritional treatment. 


\section{INTRODUCCIÓN}

La obesidad, definida por la Organización Mundial de la Salud (OMS) como un acúmulo anormal o excesivo de grasa, se ha convertido en uno de los principales problemas de Salud Pública a nivel mundial. Está asociada con un alto riesgo de enfermedad cardiovascular y algunos tipos de cáncer, lo que genera una mayor mortalidad y un aumento de morbilidad, además de un impacto perjudicial sobre la calidad de vida de quienes la padecen ${ }^{1}$, y se estima que, junto con el sobrepeso, causa la muerte de más de 3 millones de personas al año ${ }^{2}$.

En España, la prevalencia de obesidad se sitúa en población adulta en torno al $20 \%$ y más de la mitad de la población de entre 25 y 64 años presenta exceso ponderal ${ }^{2}$. Esto supone un gasto sanitario de un 7\%, lo que equivale a un coste económico de unos 2.500 millones de euros anuales ${ }^{3}$. Por ello, la OMS y otros organismos advierten sobre la necesidad urgente de la aplicación de planes de acción efectivos para corregir este problema que ya se considera pandemia mundial y que es la segunda causa de muerte prematura y evitable después del tabaco ${ }^{4}$.

A la hora de planificar un tratamiento no farmacológico para la obesidad hay que tener en cuenta un aumento en la actividad física, terapia conductual y por supuesto la dieta, siendo la combinación de todos ellos lo que mejores resultados ofrece ${ }^{5-7}$. Y aunque a día de hoy no existe consenso sobre qué terapia nutricional es la ideal para corregir este problema, diversos estudios ${ }^{8,9}$ apoyan que la dieta hipocalórica, especialmente la Dieta Mediterránea (DM) hipocalórica ${ }^{10,11}$, es el tratamiento de elección a la hora de tratar la obesidad y el síndrome metabólico.

Pero a pesar de que cada vez los tratamientos contra la obesidad son más completos, hay pacientes que no consiguen disminuir su peso corporal de forma significativa o que incluso recuperan peso durante el tratamiento ${ }^{12}$. La disminución del metabolismo basal unida a una baja adherencia al tratamiento puede hacer que muchos de los tratamientos de pérdida de peso no muestren los resultados que a priori se esperaría de ellos. Todo esto, unido al ambiente obesogénico en el que vivimos, hace muy difícil en ocasiones conseguir los objetivos marcados ${ }^{13}$.

En relación a esto, numerosos estudios y metaanálisis ${ }^{6,14,15}$ coinciden en que, a pesar de la eficacia demostrada de la dieta hipocalórica equilibrada y de la actividad física a la hora de conseguir pérdidas moderadas de peso en adultos obesos, estas pérdidas no se mantienen a largo plazo, recuperando la mayoría de los participantes el peso perdido al cabo de un año. Desafortunadamente, y a pesar de que cada vez hay más personas 
que siguen dietas de adelgazamiento bien por motivos estéticos o sanitarios, los estudios para valorar la efectividad de programas de pérdida de peso en España son relativamente escasos, por lo que se hace imprescindible el desarrollo de estrategias dirigidas a la reducción del peso corporal y la mejora de la comorbilidad asociada ${ }^{16}$.

Además, uno de los problemas más frecuentes con el que nos encontramos en los tratamientos contra la obesidad es que hay pacientes que no responden a los mismos (considerados pacientes refractarios) y no consiguen la pérdida de peso esperada. $\mathrm{A}$ pesar de que apenas existen estudios que expliquen el porqué de este fracaso, algunos autores lo achacan a la falta de tiempo en las consultas nutricionales ${ }^{17}$. Según Petermann et al., para optimizar la consulta y llevar a cabo un protocolo de "rescate", el tiempo mínimo de duración debería ser de 30 minutos por paciente, con una frecuencia quincenal o mensual para poder adaptar los tratamientos, personalizar las dietas, modificar conductas o reforzar estrategias de cara a conseguir la reducción esperada.

Por todo ello, el objetivo principal de este trabajo fue valorar el efecto de una intervención nutricional intensiva, basada en la aplicación de una DM hipocalórica personalizada, sobre el peso corporal y el perímetro de cintura en adultos refractarios a la pérdida de peso.

\section{MATERIAL Y MÉTODOS}

Tipo de estudio y muestra

Estudio cuantitativo longitudinal de 6 meses de duración.

Los participantes seleccionados provienen del estudio Predimed-PLUS, ensayo de campo multicéntrico, aleatorizado de grupos paralelos dirigido a la prevención primaria de enfermedad cardiovascular en adultos con sobrepeso y obesidad, mediante una intervención intensiva sobre el estilo de vida a base de una dieta mediterránea hipocalórica, actividad física y tratamiento conductual, que comenzó en 2013 y aún sigue activo.

Para nuestra intervención se seleccionaron 100 pacientes del nodo de León que al inicio del estudio habían sido asignados al grupo de intervención. 
Los criterios de inclusión que se tuvieron en cuenta fueron los siguientes: hombres de 55-75 años y mujeres de entre 60-75 años, cuyo IMC fuera $\geq 27$ y $<40 \mathrm{Kg} / \mathrm{m}^{2}$, que cumplieran con al menos 3 criterios de síndrome metabólico, y que no hubieran perdido al menos un $5 \%$ de su peso corporal tras un año de intervención nutricional y de promoción de actividad física moderada.

\section{Procedimiento}

Nuestro trabajo se basó en una intervención de rescate, por lo que tomamos como referencia una intervención nutricional enfocada a la reducción de masa corporal realizada anteriormente en la que no se habían conseguido los objetivos esperados. Estos objetivos habían sido la pérdida de peso y la pérdida de perímetro abdominal igual o superior al $5 \%$ en los primeros 12 meses de tratamiento.

En el estudio tomado como referencia, el $12 \%$ de la muestra había conseguido el objetivo establecido respecto a la pérdida de peso y el $21 \%$ la pérdida de perímetro abdominal, por lo que en nuestra intervención nos centramos en el grupo que no había logrado las metas marcadas.

Estos pacientes, durante 6 meses acudieron a consulta de forma individual y se les prescribió mensualmente una dieta hipocalórica personalizada basada en el patrón de Dieta Mediterránea, además de ser motivados para realizar algún tipo de actividad aeróbica moderada al menos 4 veces por semana durante 30 - 60 minutos. A todos los participantes se les entregó un podómetro al inicio de la intervención para facilitar la tarea de monitorización de la actividad física, y se les sugirió que trataran de llegar a los 10.000 pasos diarios tal y como establecen las recomendaciones actuales ${ }^{18}$. También tuvieron la oportunidad de acudir a charlas grupales una vez al mes, en las que se trabajó la motivación y se trataron diferentes temas relacionados con la alimentación y el estilo de vida saludable.

\section{Análisis estadístico}

Las características de la muestra se evaluaron mediante estadísticos descriptivos: media, desviación estándar y rango para las variables cuantitativas, y frecuencia y proporción para las variables cualitativas con los intervalos de confianza del 95\%. 
Las proporciones fueron comparadas mediante el test de Chi-cuadrado y las diferencias entre las medias se valoraron mediante la prueba de T-Student, previa evaluación de homogeneidad de varianzas según la prueba de Levene. Se consideraron valores estadísticamente significativos aquellos cuyo valor de p era menor de 0,05.

La asociación entre las variables analizadas y la variable de resultado fue establecida mediante regresión logística no condicional, calculándose los Odds Ratio (OR) con sus respectivos intervalos de confianza del $95 \%$.

Todo el análisis estadístico se llevó a cabo con el programa informático STATA v13.0. (StataCorp., 2014)

\section{Aspectos éticos}

El estudio del que provienen los participantes de la intervención fue aprobado con informe favorable por el Comité Científico y de Ética del Complejo Asistencial Universitario de León y por el Comité de Ética de Investigación de la Universidad de León, y cumple con los criterios de confidencialidad de datos de acuerdo con la Ley Orgánica de Protección de Datos 15/1999 ${ }^{19}$.

En el proceso de captación se obtuvo el consentimiento informado firmado por cada participante y fueron informados de que podían abandonar el estudio en cualquier momento sin ningún tipo de penalización o repercusión en la relación con su médico de cabecera.

\section{RESULTADOS}

La muestra seleccionada para realizar la intervención mostraba las siguientes características: 56 hombres cuya edad media al inicio del estudio era 62,7 \pm 5,1 años y 44 mujeres con una media de $66,0 \pm 4,2$ años.

El $80 \%$ de la muestra presentaba hipertensión arterial, el $24 \%$ eran diabéticos y el $17 \%$ de los participantes eran fumadores en el momento de la intervención. Respecto al nivel educativo, el $79 \%$ tenían estudios primarios o secundarios.

La media del peso de los participantes al inicio de la primera de las intervenciones se situaba en $86,1 \pm 13,7 \mathrm{~kg}$ y el perímetro de cintura en 106,5 $\pm 9,7 \mathrm{~cm}$. Al año estos valores eran $85,9 \pm 14,4 \mathrm{~kg}$ de peso medio y perímetro de cintura de 103,7 $\pm 10,4 \mathrm{~cm}$. 
En el momento de comenzar nuestra intervención el peso medio era 86,6 $\pm 14,0 \mathrm{~kg}$ y la media del perímetro abdominal se situaba en $104,7 \pm 10,9 \mathrm{~cm}$. Al finalizar el programa, los valores se situaban en $84,0 \pm 13,6 \mathrm{~kg}$ de peso medio y perímetro de cintura de 102,5 $\pm 10,6 \mathrm{~cm}$ (Figuras 1 y 2 ).

Los pacientes seleccionados para esta intervención no habían conseguido la pérdida de peso esperada en el primer tratamiento, habiendo ganado un $0,6 \pm 3,3 \%$. Tras nuestra intervención de rescate, la pérdida media fue del $-2,9 \pm 2,3 \%$, siendo esta diferencia estadísticamente significativa $(p<0,001)$.

En la tabla 1 se observa cómo el $38 \%$ de la muestra que no habían conseguido el objetivo en la primera intervención consiguieron perder más del 3\% del peso corporal tras esta, y el $26 \%$ lograron reducir su perímetro abdominal al menos un $3 \%$.

En la Tabla 2 se observan las características de los pacientes que tras la intervención de rescate consiguieron una pérdida de peso o de perímetro abdominal $\geq 3 \%$. Según nuestros resultados, los hombres tienen un $30 \%$ más de posibilidades de alcanzar la pérdida de peso esperada, los pacientes no hipertensos un $10 \%$ más de posibilidades que los hipertensos, los no diabéticos un 30\% menos que los diabéticos, y los no fumadores tres veces más posibilidades que los fumadores, a pesar de no encontrarse diferencias estadísticamente significativas $(p<0,05)$.

En cuanto al perímetro abdominal podemos decir que los hombres tienen un $10 \%$ más de posibilidades de lograr la reducción abdominal esperada, los pacientes no hipertensos un $30 \%$ más de posibilidades que los hipertensos, los no diabéticos el doble de posibilidades que los diabéticos, y los no fumadores un $20 \%$ más de posibilidades que los fumadores, a pesar de no haber encontrado diferencias estadísticamente significativas en ninguno de nuestros análisis $(p<0,05)$.

\section{DISCUSIÓN}

Los resultados obtenidos en nuestro trabajo muestran cómo una intervención intensiva, basada en una DM hipocalórica, consigue pérdidas de peso y de cintura moderadas a corto plazo, siendo la eficacia de nuestra intervención similar a la conseguida en otros estudios con diferentes estrategias de intervención.

En nuestra intervención de rescate, se consiguió una pérdida de peso media del 2,9\% a los 6 meses de tratamiento. Un 38\% de la muestra alcanzó una pérdida de peso igual 
o superior al 3\% logrando una pérdida media del 5,3\% del peso corporal. Estos resultados son más discretos que los reportados por Petermann et al., ya que en su intervención (consulta protocolizada mensual de 30 minutos de duración y 12 semanas de seguimiento) el $34 \%$ de los participantes consiguió una pérdida de peso igual o superior al $5 \%$ con una disminución media del peso de $2,7 \mathrm{~kg}{ }^{17}$. Según los autores del estudio, el tiempo elevado dedicado a cada consulta individual podría ser la clave en el logro de mejores resultados, puesto que en esta intervención se dedicaron 30 minutos a cada consulta y la media de las intervenciones nutricionales mensuales ronda los 15 minutos.

Por otra parte, nuestros resultados son más optimistas que los arrojados por los estudios de Tárraga et al. y Keyserling et al., que en intervenciones motivacionales de un año de duración consiguieron una pérdida media del $1,8 \%$ en el primero, con un $22 \%$ de la muestra alcanzando los objetivos del estudio ${ }^{20}$, y una pérdida media de $2,1 \mathrm{~kg}$ en el segundo, con un $23 \%$ de la muestra logrando el objetivo de perder más del $5 \%$ del peso corporal ${ }^{21}$. Esto puede deberse a que los mejores resultados se producen en los primeros 6 meses de intervención, ya que es en ese intervalo de tiempo cuando la motivación de los participantes es más alta. Diferentes estudios revisados coinciden en que a partir del año la mayoría de las personas recuperan parte del peso perdido ${ }^{14,15}$.

En cuanto a la reducción del perímetro abdominal, en nuestro estudio se consiguió una pérdida media del 2,1\%, logrando el $26 \%$ de la muestra una pérdida $\geq 3 \%$ en 6 meses. En este grupo la reducción del perímetro abdominal fue del 4,3\% $(4,7 \mathrm{~cm})$. Estos resultados son similares a los obtenidos en los trabajos de Petermann et al. y CarralSan Laureano et al., ya que en el primero de estos, el 34\% de los participantes consiguió en 12 semanas una reducción abdominal media de $2,9 \mathrm{~cm}{ }^{17}$, y en el segundo consiguieron una pérdida media de $6,1 \mathrm{~cm}$ en 6 meses de intervención en un hospital de día ${ }^{16}$. Por otra parte, son resultados superiores a los presentados por Tárraga et al., en los que tras una intervención motivacional de 12 meses de duración consiguieron una reducción abdominal media de 3,6 cm en los participantes.

Sin embargo, nuestros resultados son más modestos que los alcanzados por Arrebola et al., ya que en su intervención consistente en educación nutricional, apoyo psicológico y actividad física, en 6 meses de duración, los participantes lograron una reducción media de cintura de 9,4 cm ${ }^{22}$. En este caso, a pesar de que a priori el tipo de intervención es similar (restricción calórica, recomendación de actividad física y apoyo psicológico), la frecuencia de las sesiones individuales puede ser clave a la hora de conseguir mejores resultados, ya que en esta intervención las sesiones fueron quincenales y como 
ha sido comentado, un mayor contacto con el paciente se relaciona con mejores resultados.

A pesar de no haber encontrado diferencias estadísticamente significativas, otro de los datos reportados por nuestro estudio es que los hombres consiguen mayores pérdidas de peso y perímetro abdominal que las mujeres. Estos resultados coinciden con los obtenidos en un estudio llevado a cabo en la Unidad de Nutrición Clínica y Dietética del Hospital Ramón y Cajal ${ }^{23}$, en el que los hombres participantes en el estudio obtuvieron mayores pérdidas tanto de peso como de perímetro abdominal tras una intervención nutricional para la pérdida de peso de 6 meses de duración. Esto puede explicarse por la mayor tasa metabólica basal de los varones (entre un 5 y un $10 \%$ más que las mujeres), que supone entre un $60-75 \%$ del gasto energético total ${ }^{24}$.

Sin embargo, son contrarios a los resultados reportados por la intervención realizada en el Complexo Hospitalario Universitario de Vigo (CHUVI). En este estudio llevado a cabo durante dos años, las mujeres consiguieron mayores pérdidas de peso que los hombres (25). Esto podría venir dado por el hecho de que la pérdida de peso en las mujeres tiene una cierta motivación extra por cuestiones estéticas que les hace mantener la motivación en el tratamiento durante más tiempo ${ }^{26}$.

Respecto a los datos obtenidos sobre el hábito tabáquico y la reducción de masa corporal, nuestros resultados van en línea con otro artículo publicado sobre variables influyentes en la pérdida de peso ${ }^{27}$, en el que se muestra cómo las personas que no fuman o que han abandonado el hábito hace más de 4 meses tienen mayor adherencia a la dieta y por tanto consiguen una pérdida mayor de peso.

\section{CONCLUSIONES}

Una intervención intensiva, basada en una DM hipocalórica, recomendación de actividad física y charlas motivacionales, parece resultar efectiva a la hora de conseguir pérdidas moderadas de peso en pacientes refractarios a los tratamientos contra la obesidad, alcanzando en nuestro trabajo resultados similares a los publicados en otros estudios del mismo tipo.

No obstante, hay que tener en cuenta que en los estudios publicados sobre pérdida de peso en pacientes obesos existe mucha variabilidad, tanto en los protocolos de tratamiento y seguimiento como en el tiempo de duración de los programas o en las 
características de las personas evaluadas. Esto hace difícil la tarea de determinar qué tipo de protocolo es el más efectivo a la hora de tratar este problema.

Por este motivo, se hace imprescindible seguir investigando en tratamientos contra la obesidad para lograr que un mayor número de pacientes consigan los objetivos planteados de reducción de peso y mantengan esa pérdida a lo largo del tiempo.

\section{BIBLIOGRAFÍA}

1. Salas-Salvadó J, Rubio MA, Barbany M, Moreno B. Consenso SEEDO 2007 para la evaluación del sobrepeso y la obesidad y el establecimiento de criterios de intervención terapéutica. Med Clin (Barc) [Internet]. 2007 Feb 1 [cited 2018 Apr 4];128(5):184-96. Available from: https://www.sciencedirect.com/science/article/pii/S0025775307725319

2. Aranceta-Bartrina J, Pérez-Rodrigo C, Alberdi-Aresti G, Ramos-Carrera N, Lázaro-Masedo S. Prevalencia de obesidad general y obesidad abdominal en la población adulta española (25-64 años) 2014-2015: estudio ENPE. Rev Española Cardiol [Internet]. 2016 Jun 1 [cited 2017 Nov 25];69(6):579-87. Available from: http://linkinghub.elsevier.com/retrieve/pii/S0300893216001068

3. Martínez-Álvarez J. Obesidad y alimentos funcionales: ¿son efi caces los nuevos ingredientes y productos? Rev Med Univ Navarra. 2016 Oct 7;50(4):31.

4. Quiles J, Pérez C, Serra-Majem L, Román B, Aranceta J. Situación de la obesidad en España y estrategias de intervención. Rev Esp Nutr Comunitaria J Aranceta Rev Esp Nutr Comunitaria. 2008;1414(33).

5. Galán AM, Cuixart CB, Álvarez FV, Navarro Pérez J, Lobos-Bejarano JM, Ortega Sánchez-Pinilla $\mathrm{R}$, et al. Atención Primaria Recomendaciones preventivas cardiovasculares. Aten Primaria. 2012;44(1):3-15.

6. Curioni CC, Lourenço PM. Long-term weight loss after diet and exercise: a systematic review. Int J Obes [Internet]. 2005 Oct 31 [cited 2018 Jun 13];29(10):1168-74. Available from: http://www.ncbi.nlm.nih.gov/pubmed/15925949 
7. Gargallo-Fernández M, Basulto Marset J, Breton Lesmes I, Quiles Izquierdo J, Formiguera Sala X, Salas-Salvadó J. Resumen del consenso FESNAD-SEEDO: recomendaciones nutricionales basadas en la evidencia para la prevención y el tratamiento del sobrepeso y la obesidad en adultos. Endocrinol y Nutr [Internet]. 2012 Aug [cited 2018 Jun 18];59(7):429-37. Available from: http://linkinghub.elsevier.com/retrieve/pii/S1575092212001726

8. Schröder H, Marrugat J, Vila J, Covas MI, Elosua R. Adherence to the Traditional Mediterranean Diet Is Inversely Associated with Body Mass Index and Obesity in a Spanish Population. J Nutr [Internet]. 2004 Dec 1 [cited 2018 Apr 4];134(12):3355-61. Available from: https://academic.oup.com/jn/article/134/12/3355/4757515

9. Halyburton AK, Brinkworth GD, Wilson CJ, Noakes M, Buckley JD, Keogh JB, et al. Low- and high-carbohydrate weight-loss diets have similar effects on mood but not cognitive performance. Am J Clin Nutr [Internet]. 2007 Sep 1 [cited 2018 Apr 4];86(3):580-7. Available from: https://academic.oup.com/ajcn/article/86/3/580/4649430

10. Buckland G, Bach-Faig A, Serra-Majem L. Eficacia de la dieta mediterránea en la prevención de la obesidad. Una revisión de la bibliografía. Rev Española Obes. 2008;6:329-39.

11. Martínez-González MA, Salas-Salvadó J, Estruch R, Corella D, Fitó M, Ros E. Benefits of the Mediterranean Diet: Insights From the PREDIMED Study. Prog Cardiovasc Dis. 2015;58(1):50-60.

12. Hill JO, Thompson H, Wyatt H. Weight Maintenance: What's Missing? J Am Diet Assoc [Internet]. 2005 May [cited 2018 Jun 11];105(5):63-6. Available from: http://linkinghub.elsevier.com/retrieve/pii/S0002822305001586

13. Papapietro VK. Reganancia de peso después de la cirugía bariátrica. Rev Chil cirugía [Internet]. 2012 Feb [cited 2018 Jun 19];64(1):83-7. Available from: http://www.scielo.cl/scielo.php?script=sci_arttext\&pid=S0718-

40262012000100015\&lng=en\&nrm=iso\&tlng=en

14. Young MD, Callister R, Collins CE, Plotnikoff RC, Aguiar EJ, Morgan PJ. Efficacy of a gender-tailored intervention to prevent weight regain in men over 3 years: $A$ weight loss maintenance RCT. Obesity [Internet]. 2017 Jan [cited 2018 Jun 13];25(1):56-65. Available from: http://doi.wiley.com/10.1002/oby.21696 
15. Barte JCM, Ter Bogt NCW, Bogers RP, Teixeira PJ, Blissmer B, Mori TA, et al. Maintenance of weight loss after lifestyle interventions for overweight and obesity, a systematic review. Obes Rev [Internet]. 2010 [cited 2018 Jun 13]; Available from:

https://pdfs.semanticscholar.org/d1bc/0eeaddbfe20b420e673721781cb97831d8 95.pdf

16. Carral-San Laureano F, Cabo Navarro D, Ayala Ortega C, Gómez Rodríguez F, Piñero Zaldivar A, Prieto Ferrón $M$, et al. Efectividad de un programa de intervención sobre los estilos de vida en personas con obesidad atendidos en régimen de Hospital de día. Rev Calid Asist [Internet]. 2013 Sep [cited 2018 Jun 6];28(5):285-90. Available from: http://linkinghub.elsevier.com/retrieve/pii/S1134282X13000468

17. Petermann F, Durán E, Labraña AM, Celis-Morales C. Efecto de una consulta nutricional protocolizada sobre el estado nutricional y hábitos alimentarios de adultos con sobrepeso y obesidad. Rev Chil Nutr [Internet]. 2017 [cited 2018 Jun 21];44(4). Available from: https://scielo.conicyt.cl/pdf/rchnut/v44n4/0716-1549rchnut-44-04-0341.pdf

18. Tudor-Locke C, Hatano Y, Pangrazi RP, Kang Mi. How Many Steps Are Enough? Med Sci Sport Exerc [Internet]. 2008 Jul [cited 2018 Jun 14];40(Supplement):S537-43. Available from: http://www.ncbi.nlm.nih.gov/pubmed/18562971

19. Boletín Oficial del Estado. Ley Orgánica $15 / 1999$, de 13 de diciembre, de Protección de Datos de Carácter Personal . Boletín Of del Estado. 2011;289:121.

20. Tárraga ML, Rosich N, Panisello Royo JM, Gálvez Casas A, Serrano Selva JP, Rodríguez-Montes JA, et al. Eficacia de las estrategias de motivación en el tratamiento del sobrepeso y obesidad. Nutr Hosp [Internet]. 2014 [cited 2018 Jun 22];30(4):741-8. Available from: http://scielo.isciii.es/scielo.php?pid=S0212$16112014001100003 \&$ script=sci_arttext\&tlng=en

21. Keyserling TC, Samuel-Hodge CD, Pitts SJ, Garcia BA, Johnston LF, Gizlice Z, et al. A community-based lifestyle and weight loss intervention promoting a Mediterranean-style diet pattern evaluated in the stroke belt of North Carolina: the Heart Healthy Lenoir Project. BMC Public Health [Internet]. 2016 [cited 2018 Jun 22];16(732).

Available

from: 
https://www.ncbi.nlm.nih.gov/pmc/articles/PMC4975883/pdf/12889_2016_Article 3370.pdf

22. Arrebola E, Gómez-Candela C, Fernández C, Bermejo L, Loria V. Eficacia de un programa para el tratamiento del sobrepeso y la obesidad no mórbida en atención primaria y su influencia en la modificación de estilos de vida. Nutr Hosp [Internet]. 2013 [cited 2018 Jun 25];28(1):137-41. Available from: http://scielo.isciii.es/scielo.php?pid=S0212$16112013000100018 \&$ script=sci_arttext\&tlng=pt

23. Piñera MJ, Arrieta FJ, Alcaraz-Cebrián F, Botella-Carretero JI, Calañas A, Balsa JA, et al. Influencia de la pérdida de peso en la evolución clínica, metabólica y psicológica de los pacientes con sobrepeso u obesidad. Nutr Hosp [Internet]. 2012 [cited 2018 Jun 26];27(5):1480-8. Available from: http://scielo.isciii.es/scielo.php?script=sci_arttext\&pid=S021216112012000500017

24. López-Fontana CM, Martínez-González MA, Martínez JA. Obesidad metabolismo energético y medida de la actividad física. Rev Española Obes. 2003;1:29-36.

25. Carnero M, Álvarez Freire P, Molares Vila A, Álvarez González M, Carnero Gregorio O, Arias Álvarez JR, et al. Aplicación de un protocolo de tratamiento de obesidad durante 2 años. Nutr Hosp [Internet]. 2014 [cited 2018 Jun 26];29(2):300-4. Available from: http://scielo.isciii.es/scielo.php?script=sci_arttext\&pid=S021216112014000200008

26. Riobó P, Fernández-Bobadilla B, Kozarcewski M, Fernández-Moya JM. Obesidad en la mujer. Nutr Hosp. 2003;18(5):233-7.

27. Arrebola E, López-Plaza B, Koester Weber T, Bermejo López L, Palma Milla S, Lisbona Catalán A, et al. Variables predictoras de baja adherencia a un programa de modificación de estilos de vida para el tratamiento del exceso de peso en atención primaria. Nutr Hosp [Internet]. 2013 [cited 2018 Jun 26];28(5):1530-5. Available from: http://scielo.isciii.es/scielo.php?pid=S0212$16112013000500024 \&$ script=sci_arttext\&tlng=en 
Tabla 1. Pérdida de peso y cintura tras la intervención.

\begin{tabular}{|c|c|c|c|c|c|}
\hline & $\mathrm{N}$ & Media & DS & Mínimo & Máximo \\
\hline \multicolumn{6}{|l|}{ Peso } \\
\hline $\begin{array}{l}\text { Pérdida total tras intervención } \\
\text { rescate }(\%)\end{array}$ & 100 & $-2,93$ & 2,3 & $+1,1$ & $-10,3$ \\
\hline $\begin{array}{l}\text { Pérdida de peso en la } \\
\text { intervención }<3 \%\end{array}$ & 62 & $-1,49$ & 1,0 & $+1,1$ & $-2,8$ \\
\hline $\begin{array}{l}\text { Pérdida de peso en la } \\
\text { intervención } \geq 3 \%\end{array}$ & 38 & $-5,26$ & 2,0 & $-3,0$ & $-10,3$ \\
\hline Cintura & & & & & \\
\hline $\begin{array}{l}\text { Pérdida total tras intervención } \\
\text { rescate }(\%)\end{array}$ & 100 & $-2,1$ & 1,7 & $+3,4$ & $-7,6$ \\
\hline $\begin{array}{l}\text { Pérdida de cintura en la } \\
\text { intervención }<3 \%\end{array}$ & 74 & $-1,4$ & 1,1 & $+3,4$ & $-2,9$ \\
\hline $\begin{array}{l}\text { Pérdida de cintura en la } \\
\text { intervención } \geq 3 \%\end{array}$ & 26 & $-4,3$ & 1,0 & -3 & $-7,6$ \\
\hline
\end{tabular}


Tabla 2. Características de los pacientes que lograron una reducción del peso y del perímetro abdominal $\geq 3 \%$.

\begin{tabular}{lccccc}
\hline & $\mathrm{N}$ & $\mathrm{n}$ & $\%$ & OR (IC 95\%) & $\mathrm{p}$ \\
\hline $\begin{array}{l}\text { Peso } \\
\text { Sexo }\end{array}$ & & & & & \\
Mujeres & 44 & 15 & $34,1 \%$ & 1 & \\
Hombres & 56 & 23 & $41,1 \%$ & $1,3(0,6-3,1)$ & 0,476 \\
HTA & & & & & \\
Si & 80 & 30 & $37,5 \%$ & 1 & 0,837 \\
No & 20 & 8 & $40,0 \%$ & $1,1(0,4-3,0)$ & \\
Diabetes & & & & & \\
Si & 24 & 8 & $33,3 \%$ & $1,3(0,5-3,4)$ & 0,590 \\
No & 76 & 30 & $39,5 \%$ & & \\
Fumador & & & & & \\
Si & 17 & 3 & $17,7 \%$ & & \\
No & 83 & 35 & $42,2 \%$ & $3,4(0,9-12,7)$ & 0,069 \\
\hline
\end{tabular}

\section{Cintura}

Sexo

$\begin{array}{llllcl}\text { Mujeres } & 44 & 11 & 25,0 \% & 1 & \\ \text { Hombres } & 56 & 15 & 26,8 \% & 1,1(0,4-2,7) & 0,840\end{array}$

HTA.

$\begin{array}{cccccc}\text { Si } & 80 & 20 & 25,0 \% & 1 & \\ \text { No } & 20 & 6 & 30,0 \% & 1,3(0,4-3,8) & 0,649\end{array}$

Diabetes

$\begin{array}{cccccc}\text { Si } & 24 & 4 & 16,7 \% & 1 & \\ \text { No } & 76 & 22 & 29,0 \% & 2,0(0,6-6,6) & 0,238\end{array}$

Fumador

$\mathrm{Si}$

17

4

$23,5 \%$

1

No

83

22

$26,5 \%$

$1,2(0,3-4,0)$

0,799 